\title{
Public health and public law issues for the toxicological risk assessment of chemical mixtures
}

\author{
Silvio Roberto Vinceti ${ }^{1}$, Tommaso Filippini ${ }^{2}$
}

\author{
AFFILIATION \\ 1 Department of Law, University of Modena and Reggio Emilia, Modena, Italy \\ 2 Environmental, Genetic and Nutritional Epidemiology Research Center (CREAGEN), Section of Public Health, Department of Biomedical, Metabolic \\ and Neural Sciences, University of Modena and Reggio Emilia, Modena, Italy
}

CORRESPONDENCE TO

Tommaso Filippini. Environmental, Genetic and Nutritional Epidemiology Research Center (CREAGEN), Section of Public Health, Department of Biomedical, Metabolic and Neural Sciences, University of Modena and Reggio Emilia, Via Campi 287, 41125, Modena, Italy.

E-mail: tommaso.filippini@unimore.it ORCID ID: https://orcid.org/0000-0003-2100-0344

KEYWORDS

chemical mixtures, public health, risk assessment, public law, toxicology

Received: 18 November 2021, Accepted: 23 November 2021

Public Health Toxicol 2021;1(2):6

https://doi.org/10.18332/pht/144201

\section{ABSTRACT}

Humans are simultaneously exposed to a near-infinite number of substances of both nutritional and toxicological interest. While traditional risk assessment has been based on the evaluation of effects of single substances, real-life exposure is characterized by their combination into chemical mixtures. As consequence, the need to focus on substance interactions is of considerable relevance. From the public health point of view, factors able to modulate the kinetic and toxicity of chemicals need to be included in the assessment of health effects. Several European projects including SOLUTIONS, EuroMix, ECD-MixRisk and HBM4EU have been implemented aiming at the characterization of hazardous properties of chemical mixtures and the development of new approaches and tools for their risk assessment, including advanced statistical methodologies. From the public law point of view, the legislative status of mixtures is outlined in a still general way, with a specific focus on a few substances only, e.g. dietary exposure to pesticide residues. Although some advances have been made with the ongoing revision of CLP Regulation specifically focusing also on chemical mixtures, identification of mixtures and the regulation of their use raise relevant and challenging issues to be timely addressed within a collaboration of specialists from toxicology, public health, and public law. Moreover, given the transnational scope of the problem, risk assessment of chemical mixtures presents a valuable occasion for a comparative law approach.

\section{INTRODUCTION}

Mixtures, public health and toxicology

Humans are exposed to several substances of nutritional and toxicological relevance from environmental sources including food, air, and drinking water. As a consequence, our environment can be considered a source of a near-infinite number of chemical mixtures ${ }^{1}$. There is growing evidence that a thorough risk assessment should not only focus on the independent effects of one chemical but should take into consideration the joint exposure to a complex mixture of substances of nutritional and toxicological relevance to which living organisms are exposed in real life ${ }^{2}$. However, the current safety risk assessment is primarily based on understanding the effects of single substances rather than their real-life combinations into mixtures ${ }^{3}$, thus probably overlooking their combined effects ${ }^{4}$.

The human risk assessment of exposure traditionally follows the classical approach of estimating the external exposure and comparing it with health-based monitoring guidance values, generally based on evaluation of chemical concentrations in biomarkers of on-going exposure or body burden ${ }^{5}$. Many factors can modulate the kinetics and toxicity of chemicals in addition to their overall exposure, and consequently have an impact on the health effects 
associated with their exposure ${ }^{6}$, beyond their actual internal dose resulting from all sources and pathways (oral, dermal, and inhalation $)^{7,8}$. Evidence supporting that nutritional and toxic chemicals can modulate each other, their kinetics, and biological activity, further implies that interactions occurring within chemical mixtures have a potentially strong impact on health outcomes and risk assessment ${ }^{6}$.

Studies investigating the simultaneous exposure to multiple chemicals should therefore assess the interactions between these factors - which are not detected when working with one substance at a time - and bear in mind that human populations are almost always exposed to a rather large number of complex substances simultaneously. This is the reason for the growing awareness and debate over the relevance of chemical mixtures in risk assessment ${ }^{2,9}$. At European level, the Joint Research Centre (JRC) of the European Commission has started to investigate the progress in considering combined exposures to multiple chemicals in order to help translate best science into best risk assessment practice $^{10}$. For this purpose, a 2018 JRC policy brief entitled 'Something from nothing? Ensuring the safety of chemical mixtures' confronted various issues on this topic, including the specific challenges and activities to be carried out in Europe ${ }^{10}$. These include the assessment of combined exposure, especially the composition of unintentional mixtures, of combined effects through smart strategies and innovative computational tools, and of combined risks focusing among the large number of possible mixtures on priority mixtures of particular concern ${ }^{10}$. More recently, supranational agencies for risk assessment such as the European Food Safety Authority (EFSA) have highlighted and emphasized the issue of chemical mixture evaluation in the 2020-2022 programming document ${ }^{11}$. Therefore, there is clearly the need to increase our knowledge about mixture effects, designing a strategy capable to assess multichemical and multi-pathway exposures of humans and their implication on health. In addition to envisaging a stronger effort in addressing risk assessment of chemical mixtures, the aforementioned EFSA document explicitly states that: 'The integration of New Approach Methodologies (NAMs) in EFSA risk assessments will cover three complementary goals, reduction of animal testing, filling hazard information gaps for data-poor chemicals, and last but not least, moving towards more informative risk assessments, through the integration of existing (human/animal) data and NAMs, for a better mechanistic understanding of the biological interactions that lead to the hazards and risks of chemicals, both in isolation and in chemical mixtures'.

\section{METHODOLOGICAL APPROACH}

Mixture investigations: EU projects and advanced statistical tools

While several approaches have been considered and variously combined to investigate chemical mixtures ${ }^{12-15}$, few studies have directly addressed the specific challenges involved in the formal analysis of synergistic and antagonistic interactions within components of a mixture ${ }^{16-19}$. Together with the leverage and development of statistical techniques for addressing the underlying research questions, studies specifically focusing on assessing complex interactions have the potential to improve the risk assessment of single chemicals (including nutrients) within a holistic approach. To this purpose, the European Commission has promoted the implementation of studies and research consortia that assess chemical mixtures in both the environment (e.g. SOLUTIONS project ${ }^{20}$ ) and human health (e.g. EuroMix ${ }^{21}$ and ECD-MixRisk ${ }^{22}$ ). With regard to the latter category, a very promising project is the European Human Biomonitoring Initiative for Europe (HBM4EU) ${ }^{23}$ where the assessment of several chemicals has been accomplished through a specific framework that singles out priority substance group mixtures, including the collection of information on their characterization and hazardous properties, and evaluates existing evidence of human exposure to mixtures in Europe ${ }^{24}$. Similarly, the epidemiologic literature is now beginning to address lower-order interactions between components of chemical mixtures ${ }^{25}$, while statistical methodologies that use machine learning techniques for evaluating a large number of exposures can be potentially used to detect highorder interactions ${ }^{16,19,26-29}$. For example, several procedures have been recently developed to assess the overall effect of the mixture, such as the Weighted Quantile Sum (WQS) regression, elastic net regression, and Bayesian Kernel Machine Regression (BKMR), elastic net regression for interaction (INTRANET), the deletion/substitution/addition (DSA) algorithm, and boosted regression trees, some of them taking also into account the individual dose-response associations with the outcome of interest $\mathrm{t}^{13,14,16,30-34}$. These innovative approaches that assess chemical interactions have also taken into consideration the similarity of biological effects in chemicals that differ only in their potencies and in their ability to act through different modes of action or at different sites in the human body - possibly also as a consequence of their chemical form - a feature that adds further complexity to their effects and interactions ${ }^{35}$.

\section{Mixtures and public law}

The assessment and management of chemical mixtures is only partly covered by current legislation, which unfortunately focuses on single substances. In addition, the legislative status of mixtures varies greatly depending on the different types alternatively considered.

\section{Intentional mixtures}

Formulated products marketed as such and for this reason covered by the Chemical Labelling and Packaging-CLP requirements ${ }^{36}$. For intentional mixtures, the composition is known and assessments are based on the properties of the individual constituents and tests are generally carried out on the entire product. 
Unintentional mixtures

Mixtures originated from a single source where: 1 ) the composition is known and therefore assessment can be made based on knowledge of the constituents; and 2) the composition is unknown, in this case the assessment can in principle be based on tests carried out on the whole mixture or based on the single substances.

\section{Coincidental mixtures}

Mixtures of chemicals originated from multiple sources and through multiple routes.

\section{Regulations}

In the context of the REACH Regulation ${ }^{37}$, guidance has been developed concerning the assessment of multiple sources of exposure to a single substance and in specific cases to the assessment of several closely related and similarly acting substances, such as different salts of the same metal or a number of closely related derivatives of organic substances (see for example ECHA 2016, section E.3.5). While this gives some scope to assess possible adverse effects associated with known combinations, it does not address possible concerns associated with exposure to unknown mixtures. Another example of regulation of mixtures is available for pesticides with the Regulation 396/2005/EC38 on maximum residue levels of pesticides in either food and feed of plant and animal origin with the subsequent implementation of several activities of the European Food Safety Authority, including Scientific Opinions on mixture pesticides exposure ${ }^{39,40}$.

\section{DISCUSSION}

Despite these efforts, a substantial lack of legislation can be still noted ${ }^{21}$, though improvements are in progress as recently demonstrated by the public consultation to update of the CLP legislation ${ }^{41}$, focusing one of the key topics on the importance to take into account the effects of mixtures of chemicals ${ }^{42-44}$ and highlight the challenges they pose to both risk assessors and risk managers, i.e. to both toxicology and public law. From a comparative law perspective, moreover, the European legal framework for combined exposures' risk assessment is considered somehow underdeveloped when compared to those in place in the United States or Canada ${ }^{21}$.

The very American experience, however, remind us that the legal problems posed by chemical mixtures and their risk assessment are common throughout the world and that international solutions could better address them. More than forty years have passed since the controversial 'Benzene case' of July 1980, where a 5-to-4 US Supreme Court ruled that the requirement of health standards 'reasonably necessary or appropriate to provide safe or healthful employment and places of employment' was not so dire as to demand that employers apply 'the lowest technologically feasible level that will not impair the viability of the industries regulated' 45 .

\section{CONCLUSIONS}

To prohibit exposure at a certain level, regulatory agencies must demonstrate a 'significant risk' ${ }^{46}$. Forty years after the Benzene decision, there is growing awareness in the US about the need to address the ruling and somehow revise $\mathrm{it}^{47}$. For this reason, an optimal solution for both European and Non-European agencies is not simply to emulate foreign arrangement, but rather to work together and elaborate standards and common approaches that could lead to a shared legal framework for chemical mixtures ${ }^{21,48}$.

\section{REFERENCES}

1. Hernández AF, Tsatsakis AM. Human exposure to chemical mixtures: Challenges for the integration of toxicology with epidemiology data in risk assessment. Food Chem Toxicol. 2017;103:188-193. doi:10.1016/j.fct.2017.03.012

2. Tsatsakis AM, Docea A0, Tsitsimpikou C. New challenges in risk assessment of chemicals when simulating real exposure scenarios; simultaneous multi-chemicals' low dose exposure. Food Chem Toxicol. 2016;96:174-176. doi:10.1016/j.fct.2016.08.011

3. Tsatsakis AM, Kouretas D, Tzatzarakis MN, et al. Simulating real-life exposures to uncover possible risks to human health: A proposed consensus for a novel methodological approach. Hum Exp Toxicol. 2017;36(6):554-564. doi:10.1177/0960327116681652

4. Chemical mixtures: How to address the safety of combined exposures to multiple chemicals for people and the environment. European Union Science Hub, European Commission. Updated June 28, 2018. Accessed November 18, 2021. https://ec.europa.eu/jrc/en/news/chemical-mixturessafety

5. Framework for Metals Risk Assessment: EPA 120/R-07/001. United States Environmental Protection Agency; 2007. Accessed November 18, 2021. https://www.epa.gov/sites/ default/files/2013-09/documents/metals-risk-assessmentfinal.pdf

6. Cory-Slechta DA. Studying toxicants as single chemicals: does this strategy adequately identify neurotoxic risk? Neurotoxicology. 2005;26(4):491-510. doi:10.1016/j.neuro.2004.12.007

7. Choi DG, Kreikebaum F, Thomas VM, Divan D. Coordinated EV adoption: double-digit reductions in emissions and fuel use for \$40/vehicle-year. Environ Sci Technol. 2013;47(18):10703-10707. doi:10.1021/es4016926

8. Yusa V, Millet M, Coscolla C, Pardo O, Roca M. Occurrence of biomarkers of pesticide exposure in non-invasive human specimens. Chemosphere. 2015;139:91-108. doi:10.1016/j.chemosphere.2015.05.082

9. Kumari M, Kumar A. Identification of componentbased approach for prediction of joint chemical mixture toxicity risk assessment with respect to human health: A critical review. Food Chem Toxicol. 2020;143:111458. doi:10.1016/j.fct.2020.111458

10. Something from nothing? Ensuring the safety of chemical 
mixtures. European Commission, Joint Research Centre; 2018. June 8, 2018. doi: $10.2760 / 618648$

11.Programming Document 2020 - 2022: Trusted science for safe food. European Food Safety Authority (EFSA); 2019. January 31, 2020. Accessed November 18, 2021. doi:10.2805/866177

12. Taylor KW, Joubert BR, Braun JM, et al. Statistical Approaches for Assessing Health Effects of Environmental Chemical Mixtures in Epidemiology: Lessons from an Innovative Workshop. Environ Health Perspect. 2016;124(12):A227-A229. doi:10.1289/EHP547

13. Souter I, Bellavia A, Williams PL, et al. Urinary Concentrations of Phthalate Metabolite Mixtures in Relation to Serum Biomarkers of Thyroid Function and Autoimmunity among Women from a Fertility Center. Environ Health Perspect. 2020;128(6):67007. doi:10.1289/EHP6740

14. Chiu YH, Bellavia A, James-Todd T, et al. Evaluating effects of prenatal exposure to phthalate mixtures on birth weight: A comparison of three statistical approaches. Environ Int. 2018;113:231-239. doi:10.1016/j.envint.2018.02.005

15. Hamra GB, Buckley JP. Environmental exposure mixtures: questions and methods to address them. Curr Epidemiol Rep. 2018;5(2):160-165. doi:10.1007/s40471-018-0145-0

16. Lampa E, Lind L, Lind PM, Bornefalk-Hermansson A. The identification of complex interactions in epidemiology and toxicology: a simulation study of boosted regression trees. Environ Health. 2014;13:57. doi:10.1186/1476-069X-13-57

17. Sun Z, Tao Y, Li S, et al. Statistical strategies for constructing health risk models with multiple pollutants and their interactions: possible choices and comparisons. Environ Health. 2013;12(1):85. doi:10.1186/1476-069X-12-85

18. Barrera-Gómez J, Agier L, Portengen L, et al. A systematic comparison of statistical methods to detect interactions in exposome-health associations. Environ Health. 2017;16(1):74. doi:10.1186/s12940-017-0277-6

19. Hernandez AF, Buha A, Constantin C, et al. Critical assessment and integration of separate lines of evidence for risk assessment of chemical mixtures. Arch Toxicol. 2019;93(10):2741-2757. doi:10.1007/s00204-019-02547-x

20. Brack W, Altenburger R, Schüürmann G, et al. The SOLUTIONS project: challenges and responses for present and future emerging pollutants in land and water resources management. Sci Total Environ. 2015;503-504:22-31. doi:10.1016/j.scitotenv.2014.05.143

21. Rotter S, Beronius A, Boobis AR, et al. Overview on legislation and scientific approaches for risk assessment of combined exposure to multiple chemicals: the potential EuroMix contribution. Crit Rev Toxicol. 2018;48(9):796-814. doi:10.1080/10408444.2018.1541964

22. Bopp SK, Barouki R, Brack W, et al. Current EU research activities on combined exposure to multiple chemicals. Environ Int. 2018;120:544-562. doi:10.1016/j.envint.2018.07.037

23. David M, Schwedler G, Reiber L, et al. Learning from previous work and finding synergies in the domains of public and environmental health: EU-funded projects BRIDGE
Health and HBM4EU. Arch Public Health. 2020;78(1):78. doi:10.1186/s13690-020-00460-9

24. Chemical mixtures. hbm4eu.eu. Updated May, 2021. Accessed November 18, 2021. https://www.hbm4eu.eu/ the-substances/chemical-mixtures/

25. Tsatsakis AM, Docea AO, Calina D, et al. Hormetic Neurobehavioral effects of low dose toxic chemical mixtures in real-life risk simulation (RLRS) in rats. Food Chem Toxicol. 2019;125:141-149. doi:10.1016/j.fct.2018.12.043

26. Bobb JF, Valeri L, Claus Henn B, et al. Bayesian kernel machine regression for estimating the health effects of multi-pollutant mixtures. Biostatistics. 2015;16(3):493-508. doi:10.1093/biostatistics/kxu058

27. Bellavia A, Rotem RS, Dickerson AS, Hansen J, Gredal O, Weisskopf MG. The use of Logic regression in epidemiologic studies to investigate multiple binary exposures: an example of occupation history and amyotrophic lateral sclerosis. Epidemiol Methods. 2020;9(1):20190032. doi:10.1515/em-2019-0032

28. Ghaderpoori M, Kamarehie B, Jafari A, et al. Health risk assessment of heavy metals in cosmetic products sold in Iran: the Monte Carlo simulation. Environ Sci Pollut Res Int. 2020;27(7):7588-7595. doi:10.1007/s11356-019-07423-w

29. Tsatsakis A, Docea AO, Constantin C, et al. Genotoxic, cytotoxic, and cytopathological effects in rats exposed for 18 months to a mixture of 13 chemicals in doses below NOAEL levels. Toxicol Lett. 2019;316:154-170. doi:10.1016/j.toxlet.2019.09.004

30. Bellavia A, Chiu YH, Brown FM, et al. Urinary concentrations of parabens mixture and pregnancy glucose levels among women from a fertility clinic. Environ Res. 2019;168:389396. doi:10.1016/j.envres.2018.10.009

31. Bobb JF, Claus Henn B, Valeri L, Coull BA. Statistical software for analyzing the health effects of multiple concurrent exposures via Bayesian kernel machine regression. Environ Health. 2018;17(1):67. doi:10.1186/s12940-018-0413-y

32. Carrico C, Gennings C, Wheeler DC, Factor-Litvak P. Characterization of Weighted Quantile Sum Regression for Highly Correlated Data in a Risk Analysis Setting. J Agric Biol Environ Stat. 2015;20(1):100-120. doi:10.1007/s13253-014-0180-3

33. Lim M, Hastie T. Learning interactions via hierarchical grouplasso regularization. J Comput Graph Stat. 2015;24(3):627654. doi:10.1080/10618600.2014.938812

34. Molinaro AM, Lostritto K, van der Laan M. partDSA: deletion/ substitution/addition algorithm for partitioning the covariate space in prediction. Bioinformatics. 2010;26(10):1357-1363. doi:10.1093/bioinformatics/btq142

35. EFSA Scientific Committee, More SJ, Bampidis V, et al. Guidance on harmonised methodologies for human health, animal health and ecological risk assessment of combined exposure to multiple chemicals. EFSA J. 2019;17(3):e05634. doi:10.2903/j.efsa.2019.5634

36. CLP Legislation. European Chemicals Agency. Accessed November 18, 2021. https://echa.europa.eu/regulations/ clp/legislation 
37. Substances restricted under REACH. European Chemicals Agency. Accessed November 18, 2021. https://echa.europa. eu/substances-restricted-under-reach

38. Regulation (EC) No 396/2005 of the European Parliament and of the Council on maximum residue levels of pesticides in or on food and feed of plant and animal origin and amending Council Directive 91/414/EEC. Food and Agricultural Organization of the United Nation; 2005. February 23, 2005. Updated January 26, 2019. Accessed November 18, 2021. https://www.fao.org/faolex/results/details/en/c/LEXFAOC050711

39. European Food Safety Authority (EFSA), Dujardin B. Comparison of cumulative dietary exposure to pesticide residues for the reference periods 2014-2016 and 2016-2018. EFSA J. 2021;19(2):e06394. doi:10.2903/j.efsa.2021.6394

40. EFSA Panel on Plant Protection Products and their Residues (PPR). Scientific Opinion on the identification of pesticides to be included in cumulative assessment groups on the basis of their toxicological profile. EFSA J. 2013;11(7):3293. doi:10.2903/j.efsa.2013.3293

41.Vinceti SR, Docea AO, Tsitsimpikou C, Filippini T. Updating the European Union's regulation on classification, labelling and packaging of substances and mixtures (CLP): A key opportunity for consumers, workers and stakeholders with interests in the legislation and toxicology of hazardous chemicals. Toxicol Rep. 2021;8:1865-1868. doi:10.1016/j.toxrep.2021.11.011

42. Kostoff RN, Aschner M, Goumenou M, Tsatsakis A. Setting safer exposure limits for toxic substance combinations. Food Chem Toxicol. 2020;140:111346. doi:10.1016/j.fct.2020.111346

43. Margina D, Nițulescu GM, Ungurianu A, et al. Overview of the effects of chemical mixtures with endocrine disrupting activity in the context of real-life risk simulation: An integrative approach (Review). World Acad Sci J. 2019;1(4):157-164. doi:10.3892/wasj.2019.17

44. Bellavia A, James-Todd T, Williams PL. Approaches for incorporating environmental mixtures as mediators in mediation analysis. Environ Int. 2019;123:368-374. doi:10.1016/j.envint.2018.12.024

45. Industrial Union Department, AFL-CIO v American Petroleum Institute, 448 US 607 (1980). Accessed November 18, 2021. https://supreme.justia.com/cases/federal/us/448/607/
46. Stone PF. The Significant Risk Requirement in OSHA Regulation of Carcinogens: Industrial Union Department, AFL-CIO v. American Petroleum Institute. Stanford Law Review. 1981;33(3): 551-566. doi:10.2307/1228358

47. Sprinkle RH, Payne-Sturges DC. Mixture toxicity, cumulative risk, and environmental justice in United States federal policy, 1980-2016 : Why, with much known, was little done? Environ Health. 2021;20(1):104. doi:10.1186/s12940-021-00764-5

48. Carducci AL, Agodi A, Ancona C, et al. Impact of the environment on the health: From theory to practice. Environ Res. 2021;194:110517. doi:10.1016/j.envres.2020.110517

\section{CONFLICTS OF INTEREST}

The authors have completed and submitted the ICMJE Form for Disclosure of Potential Conflicts of Interest and none was reported.

FUNDING

There was no source of funding for this research.

ETHICAL APPROVAL AND INFORMED CONSENT

Ethical approval and informed consent were not required for this study.

\section{DATA AVAILABILITY}

Data sharing is not applicable to this article as no new data were created. 